Pulido Acosta, F. y Herrera Clavero, F. (2019). Estados emocionales contrapuestos como predictores del rendimientó académico en secundaria. Revista de Investigación Educativa, 37(1), 93-109.

DOI: http://dx.doi.org/10.6018/rie.37.1.289821

\title{
Estados emocionales contrapuestos como predictores del rendimiento académico en secundaria
}

\section{Opposite emotional states as a predictor of academic achievement in high school}

\author{
Federico Pulido Acosta y Francisco Herrera Clavero \\ Dpto. de Psicología Evolutiva y de la Educación, Facultad de Educación, Economía y Tecnología de Ceuta. \\ Universidad de Granada.
}

\begin{abstract}
Resumen
Este trabajo tiene como objetivo fundamental analizar los predictores de Ansiedad, Felicidad y Rendimiento Académico, considerando la edad, género, cultura y estrato socioeconómico, así como contemplar las relaciones que se pudieran dar entre las variables, para una población adolescente multicultural. Para ello, se ha contado con 811 participantes, de seis centros en los que se imparte formación perteneciente a la Etapa Secundaria. Considerando la cultura, el grupo mayoritario es el de participantes pertenecientes a la cultura-religión musulmana. Estos constituyen el $71.6 \%$ de la muestra, mientras que el $28.4 \%$ de la misma pertenecía a la culturareligión cristiana, siendo el $46.1 \%$ varones y el resto de la muestra (53.9\%) mujeres. Como instrumentos de evaluación se han empleado el Inventario de Ansiedad Estado-Rasgo para Niños (STAIC, Spielberger, 2001), un cuestionario elaborado para evaluar la Felicidad junto con las calificaciones de los estudiantes. Los resultados reflejan que los predictores de la Ansiedad son la Edad y el Género. Para la Felicidad aparecen todas las variables sociodemográficas: Edad, Cultura, Estatus y Género. Se ha halló una fuerte relación inversa entre Ansiedad y Felicidad, actuando cada una como principal predictora de la otra. En Rendimiento Académico los predictores son el estatus, la cultura/religión y la edad. No se ha halló relación estadísticamente significativa entre Ansiedad y Rendimiento Académico, pero sí en el caso de la Felicidad.

Palabras clave: ansiedad; felicidad; rendimiento académico; contexto pluricultural.

Correspondencia: Federico Pulido Acosta, feanor_fede@hotmail.com, Departamento de Psicología Evolutiva y de la Educación, Facultad de Educación, Economía y Tecnología de Ceuta. Universidad de Granada, C/ Cortadura del Valle, s/n, 51001, Ceuta.
\end{abstract}




\begin{abstract}
This work has as main objective to analyze the predictors of Anxiety, Happiness and Academic Achievement, according to age, gender, culture and socioeconomic stratum as well as the relationships that could be given between all the variables, for a multicultural adolescent sample. To make it possible we focused on 811 participants from six educational centre; $71.6 \%$ of the total were Muslims and $28.4 \%$ belonged to the Christian religion, $46.1 \%$ were male and $53.9 \%$ female. The techniques used in this survey were the State-Trait Anxiety Inventory for Children (STAIC, Spielberger, 2001), a Happiness questionnaire, as well as the students' grades. The results reflect that the predictors of Anxiety are Age and Gender. For Happiness, all the sociodemographics variables act as predictors: They are Age, Culture, Socioeconomic stratum and Gender. We found a strong and inverse relationship between Anxiety and Happiness. Each one act as the main predictor of the other. Academic Achievement predictors' are the sociodemographic factors of status, culture and age. There is no statistically significant relationship between Anxiety and Academic Achievement, but we found it between Academic Achievement and Happiness.
\end{abstract}

Keywords: Anxiety; Happiness; Emotional Intelligence; Pluricultural context.

\title{
Introducción
}

No se deben obviar las emociones y resulta fundamental en la vida saber reaccionar de manera adecuada ante ellas, ya que considerándolas como un potencial pueden transformarse en un arma muy importante con la que afrontar de manera exitosa la vida. No considerar este componente resulta algo totalmente contraproducente, puesto que supone una parte importante del éxito personal, académico y profesional.

En Pulido y Herrera (2016) se relaciona el estado de ansiedad con emociones de carácter negativo. En este sentido, consecuencias desfavorables pueden ir asociadas a este tipo de estados emocionales. La ansiedad, uno de los más conocidos, puede dificultar el adecuado desarrollo del autoconcepto (Ranoyen, Jozefiak, Wallander, Lydersen, \& Indredavik, 2014). Todo ello puede desencadenar desajustes psicosociales y enfermedades psiquiátricas. Por otro lado, Rodríguez (2010) entiende la felicidad como un estado emocional, generado por la interacción de diferentes condiciones que actúan sobre el individuo, provocando respuestas positivas. Los estados emocionales de carácter positivo, se relacionan con mayores niveles de autoestima, lo que puede favorecer el desarrollo de conductas saludables y optimismo, una mejor sensación de realización personal y adaptación del sujeto a su entorno, permitiendo su crecimiento positivo. Ambos estados emocionales pueden incidir de forma directa, sobre la propia salud, siendo un importante predictor de las determinadas enfermedades (MorenoRosset, Arnal-Remón, Antequera-Jurado, \& Ramírez-Uclés, 2016).

Se puede comprobar un descenso progresivo en el diagnóstico de problemas de ansiedad a medida que el sujeto va aumentando su edad (Ingles, La Greca, Marzo, García-López, \& García-Fernández, 2010). Otro de los elementos más relevantes dentro de este campo es su clara relación con el género, teniendo las mujeres niveles superiores de ansiedad (Pulido \& Herrera, 2016). Resulta también relevante la pertenencia a un grupo cultural sobre los estados emocionales negativos (Pulido \& Herrera, 2016). Por 
todo ello, se vuelve a remarcar la importancia del contexto socio-familiar en el que el sujeto se desarrolla, como predictor de diferencias significativas en las manifestaciones emocionales (Caballero-Domínguez, Suárez-Colorado, \& Bruges-Carbonó, 2015). Todo Lo comentado para la cultura, se relaciona de forma directa con el estatus. De la misma forma, se puede intuir cierta independencia entre el nivel de felicidad manifestado por el sujeto y su edad (Carballeira, González, \& Marrero, 2015). Por su parte, algunos autores evidencian que son los varones los que son más proclives a manifestar niveles superiores de felicidad (Quiceno \& Vinaccia, 2015). También existe influencia de la cultura sobre el desarrollo emocional y, por tanto, sobre la manifestación de diferentes estados emocionales como la felicidad (Restrepo, Vinaccia, \& Quiceno, 2011). Por todo ello, se vuelve a remarcar la importancia del contexto socio-familiar en el que el sujeto se desarrolla, como predictor de diferencias significativas en las manifestaciones emocionales (Quiceno \& Vinaccia, 2015).Todo esto se puede extender al estatus socioeconómico y cultural, dentro de este contexto.

Por otro lado, aparece el Rendimiento Académico. Desde esta perspectiva, se refiere al nivel de conocimientos y destrezas escolares exhibidos por los estudiantes. En este caso, se emplearán las calificaciones académicas como indicativo del rendimiento, siguiendo el trabajo comentado en Gómez-Castro (1986), según el cual, no existen diferencias estadísticamente significativas entre las calificaciones obtenidas en clase y las puntuaciones de diferentes pruebas empleadas para demostrar el rendimiento académico en diferentes materias.

Pulido y Herrera (2016) también establecen diferencias en función del género, que actúa como predictor del rendimiento. En este trabajo fueron las mujeres las que presentan mejores resultados que los varones. Sin embargo, la cultura es un factor mucho más determinante en las calificaciones académicas. De nuevo, Pulido y Herrera (2016) demostraron diferencias en los niveles, en dos muestras de cultural diferentes, no sólo en el rendimiento académico general, sino también en cada una de las materias. En este sentido, se mencionan las dificultades para la integración, desde el punto de vista social y educativo (Oropesa, Moreno, Pérez, \& Muñoz-Tinoco, 2014) y las diferencias de estatus socioeconómico y cultural (Cervini, Dari, \& Quiroz, 2014) como importantes justificantes de estas diferencias significativas. Por otro lado, en el contexto pluricultural en el que se pretende desarrollar el estudio, tiene mucha importancia las importantes diferencias en cuanto a la lengua materna entre musulmanes y cristianos. Otro de los elementos considerados como determinantes (Alonso \& Román, 2014; Pulido \& Herrera, 2016) es el estatus, que guarda una estrecha relación con el grupo cultural de pertenencia.

Por otro lado, se contempla también una relación entre ansiedad y rendimiento académico, dado que esta puede llegar a dificultar el desarrollo educativo, influyendo, de forma negativa en el mismo (Contreras, Espinosa, Esguerra, Haikal, \& Rodríguez, 2005), especialmente cuando aparece en niveles altos. Esta realidad permite que se pueda considerar la ansiedad moderada como elemento con una función útil, si se tiene en cuenta que genera un estado de alerta que facilita el rendimiento académico mejorando el aprendizaje mecánico.

Para concluir, se considerarán las relaciones descritas entre la felicidad y este indicador del ámbito académico. Desde la perspectiva de la relación entre estados 
emocionales y rendimiento académico, se establecen claras relaciones entre el bienestar psicológico y el desempeño académico (Salanova, Martínez, Bresó, Llore, \& Grau, 2005), incidiendo en la importancia que tienen los trabajos en Psicología Positiva, centrada en el desarrollo óptimo y los aspectos positivos. Salanova et al. (2005) encontraron relaciones significativas entre bienestar emocional y (incluyendo la felicidad) y rendimiento académico. Estos autores demostraron que los niveles más altos en rendimiento se relacionan con mayores niveles de autoeficacia, satisfacción y felicidad. Cuando se realiza este análisis considerando el rendimiento, se constató que a mayor rendimiento en el pasado, niveles más altos de bienestar psicológico en el futuro. Por contra, cuanto más bajo son los niveles de bienestar, peor rendimiento se obtiene en el futuro. Por ello, tiene relevancia la combinación de cansancio emocional, el descenso del interés en el desarrollo de actividades académicas y una deficiente motivación (Schaufeli \& Bakker, 2005).

Considerando todo lo mencionado, se presenta el estudio llevado a cabo con una muestra de alumnos escolarizados en Institutos de Enseñanza Secundaria en la ciudad de Ceuta, donde conviven principalmente personas de cultura cristiana y musulmana. Los objetivos del trabajo han sido conocer cuáles son los predictores de ambos estados emocionales (ansiedad y felicidad) así como los del Rendimiento Académico, en función de la edad, género, cultura y estrato socioeconómico. También se pretende contemplar las relaciones que se pudieran dar entre las mencionadas variables, actuando la una como predictora de la otra, para una población adolescente multicultural. De esta manera, se pretende determinar si estos estados emocionales, considerando los instrumentos que se emplearán, aparecen como elemento a tener en cuenta en la mejora del Rendimiento Académico. Por otra parte, llevar a cabo este estudio en un contexto pluricultural ha permitido considerar el comportamiento de las variables en dos grupos culturales con características diferentes.

\section{Método}

La muestra estuvo compuesta por 811 participantes, repartidos entre seis centros en los que se imparte formación perteneciente a la Etapa Secundaria. La distribución de la muestra, considerando las variables sociodemográficas, aparece resumido en la tabla 1 .

Tabla 1

Muestra en función de variables sociodemográficas

\begin{tabular}{ccccc}
\hline Variables sociodemográficas & \multicolumn{3}{c}{ Media descriptiva } \\
\hline \multirow{2}{*}{ Edad } & $\mathrm{N}=811$ & Media & D.T. & Rango \\
& & 15.39 & 4.46 & $12-47$ \\
\hline \multirow{3}{*}{ Cultura/Religión } & Cristianos & $\mathrm{N}$ & $\%$ \\
& Musulmanes & 221 & 28.4 \\
& & 556 & 71.6 \\
\hline
\end{tabular}




\begin{tabular}{rrrc}
\hline Género & Varones & 363 & 46.1 \\
& Chicas & 424 & 53.9 \\
\hline & Bajo & 25 & 3.2 \\
& Medio-Bajo & 173 & 22.3 \\
\multirow{2}{*}{ Estatus } & Medio & 521 & 67.2 \\
& Alto & 56 & 7.2 \\
\hline
\end{tabular}

Con respecto a la primera variable de estudio (se entiende por variable de estudio las puntuaciones totales que aglomeran los diferentes factores en los que esta se divide), la Ansiedad, se utilizó el Inventario de Ansiedad Estado-Rasgo para Niños (STAIC, Spielberger, 2001). El STAIC queda conformado por 40 ítems, con cuatro posibilidades de respuesta cada uno. Su consistencia interna, medida a través de la prueba de Cronbach, fue de .934. Esto también queda demostrado por la prueba de dos mitades de Spearman-Brown. Este coeficiente fue de .781 para el cuestionario, incluyendo todos los ítems. En el apartado relacionado con la varianza factorial se emplearon diferentes Análisis Factoriales Confirmatorios (AFC). En este sentido, los factores obtenidos fueron 6: Estados emocionales positivos ( $31.53 \%$ de la varianza explicada), Preocupaciones y dificultades $(9.487 \%)$, Estados emocionales negativos (4.35\%), Rasgos emocionales negativos (3.98\%), Sensaciones de ansiedad (3.31\%) y Miedo (2.99\%). Entre todas las categorías se consigue una varianza total explicada del $55.676 \%$.

Para la segunda variable de estudio, la Felicidad, se usó un instrumento conformado por 40 ítems a los que se contesta con una escala tipo Lickert de 4 puntos. En este caso, los resultados que hacen referencia a la consistencia interna del instrumento dan una fiabilidad, establecida por un de Cronbach de .902 y una prueba de dos mitades de Spearman-Brown de .833. En el apartado relacionado con la varianza factorial (AFC), los factores obtenidos también fueron 6: Satisfacción ante la vida (27.67\% de la varianza explicada), Condiciones en la vida (10.04\%), Cambios en la vida (4.17\%), Condiciones positivas en la vida (3.49\%), No Cambios en la vida (3.32\%) e Instituto (3.06\%). Entre todos los factores suman una varianza total explicada del $51.78 \%$.

Finalmente para la tercera variable de estudio, se emplearon las calificaciones, como medida del Rendimiento Académico, siguiendo el estudio de Gómez-Castro (1986). En este caso, se usaron las calificaciones en 6 materias diferentes. Las materias consideradas fueron Lengua Castellana, Matemáticas, ciencias Sociales, ciencias Naturales, Lengua Extranjera (Inglés) y Religión. Finalmente, para el Rendimiento Total se halló la media de las calificaciones en cada una de las áreas comentadas.

Una vez construida la base de datos, se comenzó por la comprobación de las características de la muestra, contemplando su distribución en función de las diferentes variables sociodemográficas (fueron edad, género, cultura y estatus). Se continuó con el análisis regresional (regresiones múltiples paso a paso o "stepwise"). Como variables criterio (dependientes) se utilizaron cada una de las variables de estudio (Ansiedad, Felicidad y Rendimiento Académico). Dentro de estas, para hacer el análisis más específico y detallado se consideraron también cada una de las áreas académicas (especificadas en el párrafo anterior). En el análisis regresional de cada una de ellas, se emplearon las variables sociodemográficas mencionadas como predictoras 
(independientes). Además de las variables sociodemográficas, se incluyeron (como independientes) aquellas variables de estudio que no se consideraban como variable criterio (dependiente) y las áreas académicas (Rendimiento) o factores (Ansiedad y Felicidad) que las conformaban. Para determinar la función de regresión se consideró el último paso y, dentro de él, en el orden de entrada de las variables en la función de regresión, el valor de cada variable y su significación. Para conocer el porcentaje de la variación de la variable criterio (dependiente) que depende de las predictoras (independientes) se especifican el coeficiente de correlación lineal de Pearson $(R)$, el coeficiente de determinación múltiple $\left(R^{2}\right)$ y la varianza explicada de la variable más importante (la que más porcentaje explica, la cual viene determinada por el coeficiente de correlación estandarizado - $\beta$-). Para reforzar los resultados obtenidos, se concluyó con la prueba $t$ de Student de diferencias de medias para muestras independientes para aquellas variables dicotómicas, mientras que para el resto se empleó el análisis de la varianza (ANOVA de un factor). El análisis completo que ha sido posible gracias al programa informático Statistical Package for Social Sciences (SPSS 20, 2011).

\section{Resultados}

La variable Felicidad Total se encuentra relacionada con tres factores de la Ansiedad: Estados emocionales positivos, Preocupaciones y dificultades y rasgos emocionales negativos, actuando estas de forma inversamente proporcional. También se encuentra relacionada con la media del Rendimiento Académico, la Cultura, el Estatus, así como el Miedo, único factor de la Ansiedad que alcanza valores positivos. Entre todas las predictoras explican el $54.5 \%$ de la varianza total explicada por este modelo $\left(R^{2}=.545\right)$. Una mayor fuerza es ejercida por el factor Estados emocionales positivos, que alcanza un coeficiente de regresión estandarizado de 39.5\% ( $\beta=.395)$. El Estatus, la Cultura/ Religión y la media de Rendimiento Académico actúan de manera positiva. Esto indica que son los musulmanes y los que pertenecen a un estatus superior, los que alcanzan calificaciones más altas (ver tabla 2).

Tabla 2

Coeficientes de la regresión para la Felicidad

\begin{tabular}{lcccc}
\hline \multicolumn{1}{c}{ Variables } & $\mathbf{B}$ & $\boldsymbol{\beta}$ & $\mathbf{t}$ & $\mathbf{P}$ \\
\hline Constante General & 74.633 & & 19.088 & .000 \\
Estados Positivos & -1.248 & -.395 & -12.060 & .000 \\
Rasgos Negativos & -1.551 & -.364 & -9.488 & .000 \\
Media Rendimiento & 1.321 & .121 & 3.974 & .000 \\
Cultura/Religión & 4.502 & .127 & 4.477 & .000 \\
Estatus & 1.966 & .074 & 2.576 & .010 \\
Preocupaciones & -.236 & -.095 & -2.582 & .010 \\
Miedo & .675 & .077 & 2.176 & .030 \\
\hline & $\mathrm{R}=.738$ & $\mathrm{R}^{2}=.545$ & $\mathrm{~F}=89.859$ & $\mathrm{p}=.000$ \\
\hline
\end{tabular}


Felicidad Total $=74.633-1.248($ Estados Positivos $)-1.551($ Rasgos negativos $)+1.321$ (Media Rendimiento) +4.502 (Cultura) +1.966 (Estatus) -.236 (Preocupaciones) +.675 (Miedo)

La variable Ansiedad se relaciona con la Felicidad Total (junto a dos de los factores que conforman esta variable, siendo Condiciones de vida negativas y necesidad de Cambios en la vida), la Edad y el Género. El coeficiente de correlación múltiple ( $\mathrm{R}=.741)$ señala una intensa relación entre la Ansiedad y las variables predictoras, explicando, el conjunto de todas ellas, el $54.9 \%$ de la varianza explicada por el modelo $\left(\mathrm{R}^{2}=.549\right)$. El predictor más importante es el factor Condiciones negativas en la vida, el cual actúa de forma inversamente proporcional (como la Felicidad Total y el otro factor de la misma). Su coeficiente de regresión estandarizado demuestra esta influencia, explicando el 35.3\% de la varianza en el modelo ( $\beta=.353$ ). El resto (Edad y Género) lo hace en sentido positivo (ver tabla 3).

$$
\begin{gathered}
\text { Ansiedad Total }=90.976-.260(\text { Felicidad Total })-1.293(\text { Condiciones Negativas }) \\
+2.150(\text { Edad })+5.410(\text { Género })-.974(\text { Cambios vida })
\end{gathered}
$$

Tabla 3

\begin{tabular}{|c|c|c|c|c|c|}
\hline Variables & & B & $\beta$ & $t$ & $\mathbf{P}$ \\
\hline Constante General & & 90.976 & & 19.153 & .000 \\
\hline Felicidad Total & & -.260 & -.215 & -4.323 & .000 \\
\hline Condiciones Negativas & & -1.293 & -.353 & -7.516 & .000 \\
\hline Edad & & 2.150 & .199 & 6.913 & .000 \\
\hline Género & & 5.410 & .139 & 4.984 & .000 \\
\hline Cambios en mi vida & & -.974 & -.139 & -4.222 & .000 \\
\hline $\mathrm{R}=.741$ & $\mathrm{R} 2=.549$ & $\mathrm{~F}=104.587$ & & & \\
\hline
\end{tabular}

Coeficientes de la regresión para la Ansiedad Total

Cada uno de ambos estados emocionales actúa como principal predictor del otro (de forma directa o bien a través de sus factores), actuando ambos de forma inversamente proporcional. Para confirmar la relación entre ambos estados emocionales, se realizó un ANOVA, considerando la variable Ansiedad (puntuación total) como factor y la Felicidad y sus factores como dependientes. Vuelven a aparecer diferencias estadísticamente significativas en Felicidad Total $(p=.000)$ y en las categorías que pertenecen a esta variable, como es el caso de la Satisfacción con la vida $(p=.000)$, las Condiciones de vida negativas $(p=.000)$, los Cambios en la vida $(p=.000)$, las Condiciones de vida positivas $(p=.000)$ y las emociones que genera el Instituto $(p=.000)$. En este caso, con respecto a la variable Felicidad, se observa un descenso claro y progresivo en sus puntuaciones (y las de sus factores) a medida que se sube en las de Ansiedad.

En el caso de la variable Felicidad, el primer predictor es la cultura, dentro de las variables sociodemográficas. Para reforzar los resultados encontrados, se procedió a la realización de la prueba t de Student, que vuelve a confirmar esta relación con la Felicidad. Así se pueden afirmar las diferencias estadísticamente significativas en la 
variable Felicidad Total ( $p=.001)$ y en algunas de las categorías que pertenecen a esta variable (Satisfacción con la vida $-p=.001-$, Condiciones de vida negativas $-p=.007-\mathrm{y}$ Cambios en la vida $-p=.003-)$. En este caso, son los cristianos (78.34) los que presentan una media menor a los musulmanes (82.56).

Como predictor de la Felicidad también se encuentra el estatus socio económico y cultural. En este sentido, además de actuar como predictor, se observa una distribución organizada, ya que, por media, los niveles de ansiedad van subiendo a medida que se desciende en el estatus. Los niveles más altos aparecen en el estatus alto (85.55 alto y medio 82.67), con puntuaciones cercanas. El nivel medio-bajo (77.78) es el siguiente. Después aparecería el estatus bajo (69.52). Para determinar si estas diferencias eran significativas se usó la prueba ANOVA. Las diferencias vuelven a ser estadísticamente significativas en Felicidad (Total $p=.000$ ), así como tres de sus factores (Satisfacción con la vida $-p=.000-$, Condiciones de vida negativas $-p=.001-\mathrm{y}$ Condiciones de vida positivas $-p=.000-)$.

La primera variable sociodemográfica que actúa como predictora de la Ansiedad es la Edad. Para confirmar su relación, se empleó el Análisis de la Varianza (ANOVA de un factor), con la variable Edad como independiente. Esta prueba confirmó la influencia del Edad sobre la Ansiedad Total $(p=.000)$, lo mismo que ocurrió en los factores relacionados con esta variable (Estados emocionales positivos $-p=.000-$, Preocupaciones y dificultades $-p=.000-$, Estados emocionales negativos $-p=.000-$, Rasgos emocionales negativos $-p=.000-$, Sensaciones de Ansiedad $-p=.000-$ y Miedo $-p=.000-$ ). En todas ellas se encontraron diferencias estadísticamente significativas. En este caso, resulta relevante que el grupo en el que se registran los mayores niveles de ansiedad es precisamente en el de los alumnos de 17-18 años (con una media de 51.12).

En función del género, que actúa como variable predictora de la Ansiedad, se puede ver que las chicas tienen un nivel más alto de que los varones, tal y como reflejan las medias de ambos (32.60 los hombres y 41.83 las mujeres). Esta realidad se repite también en cada uno de los factores que integran esta variable. Una vez hecha esta primera aproximación, se confirmó a través de la prueba t de Student. Considerando el análisis de esta prueba, las diferencias vuelven a ser estadísticamente significativas en Ansiedad Total $(p=.000)$, en los Estados emocionales positivos $(p=.000)$, Preocupaciones y dificultades $(p=.000)$, los Estados emocionales negativos $(p=.000)$, los Rasgos emocionales negativos $(p=.000)$, Sensaciones de Ansiedad $(p=.000)$ y el Miedo $(p=.000)$. En todos los casos, los varones presentan puntuaciones más bajas.

La media del Rendimiento Académico (media de calificaciones) está bajo la influencia de varias variables. De todas ellas, la más determinante es el Estatus, cuyo coeficiente de regresión estandarizado es del 14.2\% $(\beta=.142)$. Esta variable actúa de manera directamente proporcional. Por otra parte, también ejercen influencia los factores de la Felicidad, Instituto y No cambios en la vida, la Cultura/Religión y la Edad. De ellas, la Cultura/Religión y la Edad actúan de forma inversamente proporcional. Esto indica que a medida que se asciende en Edad y entre los sujetos pertenecientes a la cultura musulmana, es inferior la media del Rendimiento. Los factores de la Felicidad actúan de manera positiva. El resto de variables quedan excluidas, explicando entre todas las predictoras el $37.7 \%$ de la varianza total $\left(\mathrm{R}^{2}=.377\right)$. La influencia de estas variables es intensa, tal y como indica el coeficiente de correlación $(\mathrm{R}=.614)$ (ver tabla 4). 
Media Rendimiento $=1.588+.348$ (Estatus) +.096 (Instituto $)+.053$ (No cambios vida) -.276 (Cultura/Religión) - .042 (Edad) + .030 (Condiciones positivas)

Tabla 4

Coeficientes de la regresión para la Media de Rendimiento Global

\begin{tabular}{|c|c|c|c|c|}
\hline Variables & B & $\beta$ & $t$ & $\mathbf{P}$ \\
\hline Constante General & 1.588 & & 2.994 & .003 \\
\hline Estatus & .348 & .142 & 4.204 & .000 \\
\hline Instituto & .096 & .097 & 2.688 & .007 \\
\hline No cambios en vida & .053 & .099 & 2.999 & .003 \\
\hline Cultura/Religión & -.276 & -.085 & -2.516 & .012 \\
\hline Edad & -.042 & -.082 & -1.998 & .046 \\
\hline Condiciones Positivas & .030 & .071 & 1.987 & .047 \\
\hline $\mathrm{R}=.614$ & $\mathrm{R}^{2}=.377$ & & & \\
\hline
\end{tabular}

Para conocer el Rendimiento en la materia de Lengua Castellana y Literatura, los resultados que se presentan, indican algo similar a lo comentado en el Rendimiento general, ya que se repiten muchas de las variables influyentes antes comentadas. La principal variable predictora es la Edad, con un $18.1 \%$ de la varianza total explicada. Además se repiten el Estatus, la Cultura/Religión, así como el factor de la Felicidad, Cambios en la vida. Este factor es directamente proporcional a las puntuaciones en la materia de Lengua Castellana. La Edad actúa de manera inversamente proporcional. Eso mismo ocurre con la Cultura/Religión. El conjunto de todas ellas da cuenta del 27.3\% de la varianza en cuanto a la variable Rendimiento en Lengua Castellana $\left(R^{2}=.273\right)$ (ver tabla 5).

$$
\begin{gathered}
\text { Rendimiento Lengua Castellana }=1.342-.331(\text { Edad })+.331 \text { (Estatus) } \\
+.076 \text { (No cambios vida })-.342(\text { Cultura/Religión })
\end{gathered}
$$

Tabla 5

\begin{tabular}{|c|c|c|c|c|}
\hline Variables & B & $\beta$ & $\mathbf{t}$ & $\mathbf{P}$ \\
\hline Constante General & 1.342 & & 2.310 & .021 \\
\hline Edad & -.182 & -.181 & -4.977 & .000 \\
\hline Estatus & .331 & .110 & 3.106 & .002 \\
\hline No cambios en vida & .076 & .115 & 3.263 & .001 \\
\hline Cultura/Religión & -.342 & -.086 & -2.397 & .017 \\
\hline $\mathrm{R}=.522$ & $\mathrm{R}^{2}=.273$ & & & \\
\hline
\end{tabular}

Coeficientes de la regresión para el Rendimiento en Lengua Castellana

El Rendimiento Académico en Matemáticas, tiene como principal variable predictora el factor Instituto (perteneciente a la Felicidad). Su coeficiente de regresión estandarizado es $\beta=.117$. De esta manera, se puede predecir partiendo del Estatus, la Edad y las 
Condiciones de vida positivas (Felicidad). De todas ellas, sólo la Edad actúa de manera inversamente proporcional, siendo el resto valores positivos. El conjunto de todas las variables predictoras da cuenta del $22.9 \%$ de la varianza, en cuanto a la variable Rendimiento en Matemáticas $\left(\mathrm{R}^{2}=.229\right)$. El Rendimiento en esta materia decrece con la edad, de la misma manera que lo hace ante los sujetos con puntuaciones bajas en los factores Instituto y Condiciones de vida positivas (ver tabla 6).

$$
\begin{gathered}
\text { Rendimiento Matemáticas }=.819+.154 \text { (Instituto })+.353 \text { (Estatus) } \\
-.087(\text { Edad })+.063(\text { Condiciones positivas })
\end{gathered}
$$

Tabla 6

Coeficientes de la regresión para el Rendimiento en Matemáticas

\begin{tabular}{lcccc}
\hline \multicolumn{1}{c}{ Variables } & $\mathbf{B}$ & $\boldsymbol{\beta}$ & $\mathbf{t}$ & $\mathbf{P}$ \\
\hline Constante General & .819 & & 1.215 & .225 \\
Instituto & .154 & .117 & 3.018 & .003 \\
Estatus & .353 & .108 & 2.909 & .004 \\
Edad & -.087 & -.116 & -3.223 & .001 \\
Condiciones Positivas & .063 & .112 & 2.806 & .005 \\
\hline \multicolumn{2}{c}{$\mathrm{R}=.479$} & $\mathrm{R}^{2}=.229$ & $\mathrm{~F}=19.774$ & $\mathrm{p}=.000$ \\
\hline
\end{tabular}

Las calificaciones obtenidas en la materia de ciencias Sociales también se pueden predecir. Queda determinado por la variable Estatus, variable de mayor importancia, por su mayor relación con el coeficiente de correlación múltiple. Esta valor alcanza un porcentaje del 14\% $(\beta=.140)$. El Estatus actúa de manera directamente proporcional, siendo superiores las puntuaciones ante estatus superiores. La Edad actúa de manera negativa, subiendo las calificaciones a medida que se desciende en edad. El factor que implica la No necesidad de provocar cambios en la vida (Felicidad) actúa de manera positiva, aumentando sus niveles en las puntuaciones más altas. El conjunto de todas ellas dan cuenta del $23.1 \%$ de la varianza en cuanto a la variable dependiente $\left(R^{2}=.231\right)$ (ver tabla 7$)$.

$$
\text { Rendimiento Sociales }=.886+.445(\text { Estatus })-.138(\text { Edad })+.063(\text { No cambios vida })
$$

Tabla 7

Coeficientes de la regresión para el Rendimiento en Ciencias Sociales

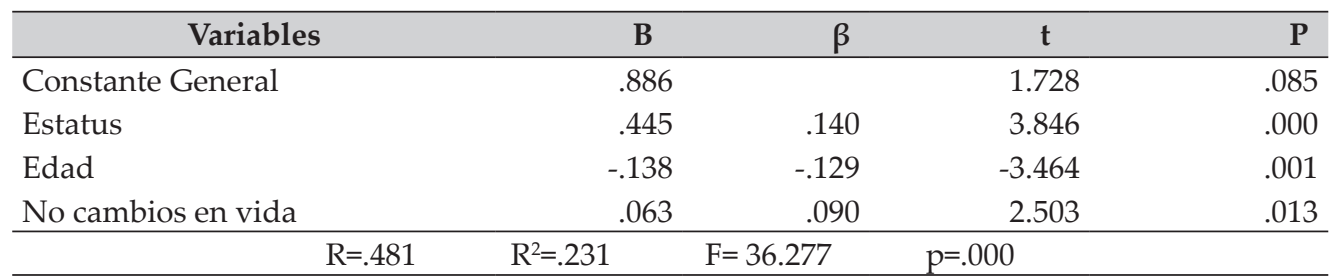


Para el Rendimiento de la materia de ciencias Naturales, de nuevo el Estatus tiene el mayor peso, representando un porcentaje mayor de la varianza total explicada $(11.3 \%$, como informa su coeficiente de regresión estandarizado $-\beta=.113-)$. Además de esta aparece, de nuevo, el factor Instituto (Felicidad), actuando de manera positiva (a medida que ascienden los niveles de satisfacción con el mismo, aumentan también los niveles en esta materia). La Edad es la única variable que adopta un valor negativo. El conjunto de todas las variables predictoras reflejan el $26.9 \%$ de la varianza total de la variable Rendimiento en Naturales $\left(\mathrm{R}^{2}=.269\right)$ (ver tabla 8$)$.

$$
\text { Rendimiento Naturales }=.618+.142(\text { Instituto })+.361(\text { Estatus })-.061(\text { Edad })
$$

Tabla 8

Coeficientes de la regresión para el Rendimiento en Ciencias Naturales

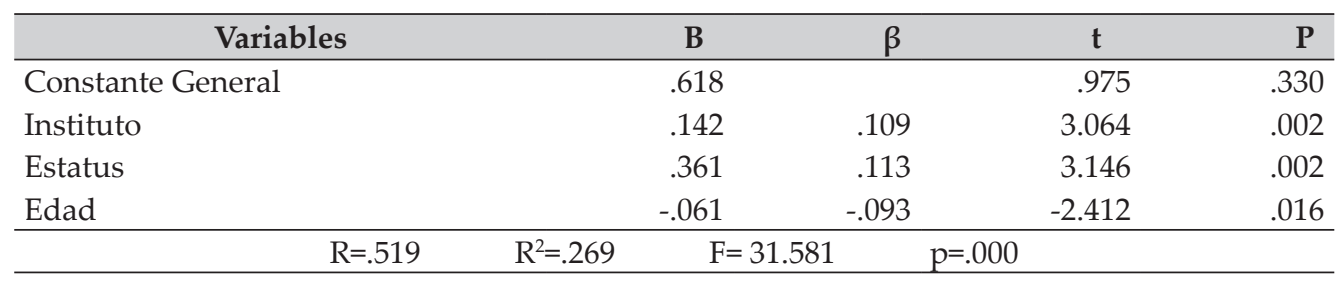

La variable Rendimiento en Inglés queda determinada por las variables Estatus, cuyo coeficiente de regresión estandarizado alcanza el nivel más alto para este modelo $(\beta=.109)$, siendo el que explica con más intensidad la relación con la variable dependiente. Como en casos anteriores, actúa de forma positiva (directamente proporcional). El factor de la Felicidad, No provocar cambios en mi vida también adopta un valor positivo. El resto de variables quedan eliminadas. El conjunto de todas las variables predictoras dan cuenta del $18.9 \%$ de la varianza en cuanto a la variable Rendimiento en la asignatura Inglés $\left(\mathrm{R}^{2}=.189\right)$ (ver tabla 9).

Rendimiento Inglés $=.617+.369$ (Estatus) $+.054($ No cambios en vida $)$

Tabla 9

Coeficientes de la regresión para el Rendimiento en Inglés

\begin{tabular}{lcccr}
\multicolumn{1}{c}{ Variables } & $\mathbf{B}$ & $\boldsymbol{\beta}$ & $\mathbf{t}$ & $\mathbf{P}$ \\
\hline Constante General & .617 & & 1.129 & .259 \\
Estatus & .369 & .109 & 2.944 & .003 \\
No cambios en vida & .054 & .072 & 1.970 & .049 \\
\hline \multicolumn{1}{r}{$\mathrm{R}=.435$} & $\mathrm{R}^{2}=.189$ & $\mathrm{~F}=28.098$ & $\mathrm{p}=.000$ & \\
\hline
\end{tabular}

Para finalizar este apartado de análisis regresional se concluye con el Rendimiento en la última área, que es Religión. El Estatus socioeconómico y cultural es la principal 
variable predictora, con un coeficiente de regresión estandarizado del $14.5 \%(\beta=.145)$ de la varianza total en cuanto a la nota en Religión. El Estatus adquiere un valor positivo (los niveles aumentan a medida que se asciende en el Estatus). Lo mismo ocurre con el factor de la Felicidad Instituto, que también adopta valores positivos (como en el resto de materias anteriormente analizadas). Para la Edad los valores son, sin embargo, negativos indicando un descenso del rendimiento a medida que se asciende en edad cronológica. Lo mismo que ocurre con la variable Cultura/Religión, lo que indica que son los cristianos los que tienen puntuaciones más altas. Finalmente, para el factor de la Felicidad (No cambios en la vida) los valores actúan de manera directamente proporcional. El conjunto de todas las variables predictoras dan cuenta del $22.2 \%$ de la varianza total explicada en la calificación en la materia de Religión $\left(\mathrm{R}^{2}=.222\right)$ (ver tabla 10).

$$
\begin{gathered}
\text { Rendimiento Religión }=3.589+.434 \text { (Estatus })+.165 \text { (Instituto })-.079 \text { (Edad) } \\
+.058(\text { No cambios vida })-.295(\text { Cultura/Religión })
\end{gathered}
$$

Tabla 10

\begin{tabular}{|c|c|c|c|c|}
\hline Variables & B & $\beta$ & $t$ & $\mathbf{P}$ \\
\hline Constante General & 3.589 & & 5.069 & .000 \\
\hline Estatus & .434 & .145 & 3.923 & .000 \\
\hline Instituto & .165 & .137 & 3.722 & .000 \\
\hline Edad & -.079 & -.128 & -3.423 & .001 \\
\hline No cambios en vida & .058 & .088 & 2.426 & .016 \\
\hline Cultura/Religión & -.295 & -.075 & -2.012 & .045 \\
\hline $\mathrm{R}=.471$ & $\mathrm{R}^{2}=.222$ & $\mathrm{~F}=24.527$ & $\mathrm{p}=.000$ & \\
\hline
\end{tabular}

Coeficientes de la regresión para el Rendimiento en Religión/Ciudadanía

Considerando las variables predictoras para el Rendimiento, se inicia la descripción del comportamiento de la muestra de alumnos de Secundaria en función de la variable sociodemográfica Estatus. De esta manera, se observa que las calificaciones aumentan progresivamente a medida que se asciende en el estatus. Es en el estatus alto en el que aparecen niveles superiores (7.08 de media). Para el medio, la calificación media es de 6.8. Las calificaciones descienden bastante para los otros dos grupos. El medio bajo (6.11) y el bajo (5.33) presentan peores resultados. Una vez hecha esta primera aproximación, el Análisis de la Varianza (ANOVA de un factor), demostró diferencias estadísticamente significativas en la Media general $(p=.000)$, así como en las áreas específicas: Lengua Castellana $(p=.000)$, Matemáticas $(p=.000)$, Sociales $(p=.000)$, Inglés $(p=.000)$, Naturales $(p=.000)$ y Religión $(p=.000)$.

En segundo lugar, aparece la edad, variable que también actúa como predictora en la mayoría de casos. El Análisis de la Varianza (ANOVA de un factor), con la variable Edad como independiente y el resto de las variables como dependientes, muestra diferencias estadísticamente significativas en la Media general $(p=.000)$. Lo mismo ocurre con las materias específicas: Lengua Castellana $(p=.000)$, Matemáticas $(p=.000)$, Sociales $(p=.019)$, Inglés $(p=.000)$, Naturales $(p=.000)$ y Religión $(p=.000)$. 
Si se tiene en cuenta la Media del Rendimiento, en función de la cultura/religión, se puede decir que las calificaciones son claramente superiores entre los cristianos. La media de los cristianos es de 7.15, mientras que la de los musulmanes es de 6.45. Por lo tanto, se puede ver que los pertenecientes al grupo "cristianos" tienen un Rendimiento Académico superior, realidad que se repite en cada una de las materias (en Lengua Castellana 6.92 los cristianos y 6.17 los musulmanes, en Matemáticas, 6.48 frente a 5.93, en ciencias Sociales, los musulmanes 6.7, mientras que los cristianos 7.33, en Inglés, los cristianos 6.96 y los musulmanes 6.20, en ciencias 7.09 los cristianos y 6.32 los musulmanes y en Religión/Ciudadanía la población de cultura cristiana 8.09 y los musulmanes 7.41). Esta realidad queda confirmada por la prueba t de Student, dado que las diferencias fueron estadísticamente significativas en la Media general $(p=.000)$, en Lengua Castellana $(p=.000)$, Matemáticas $(p=.001)$, Sociales $(p=.033)$, Inglés $(p=.000)$, Naturales $(p=.000)$ y Religión $(p=.000)$. En todos los casos, son los musulmanes los que obtienen resultados más bajos.

Se finaliza el apartado de los resultados, considerando el Rendimiento Académico, en función de la variable Ansiedad. En este sentido, ni los totales ni ninguno de los factores actuó como predictor. Esto queda confirmado a través de la prueba ANOVA, dado que las diferencias son significativas exclusivamente en el Rendimiento en Matemáticas ( $p=.014)$, descendiendo las calificaciones a medida que se asciende en los niveles de ansiedad. Sin embargo, para la Media de Rendimiento Académico ( $p=.083$, así como para el resto de las materias (Lengua Castellana $-p=.277-$, Sociales $-p=.401-$, Inglés $-p=.219-$, Naturales $-p=.123-\mathrm{y}$ Religión $-p=.569-)$, las diferencias no fueron estadísticamente significativas. Si se considera la Felicidad (a través de sus factores), sin embargo, sí se puede hablar de variable predictora. Esto queda confirmado por el Análisis de la Varianza (ANOVA de un factor). De esta manera, se encontraron diferencias estadísticamente significativas en la Media general $(p=.000)$, así como en las áreas Lengua Castellana $(p=.010)$, Matemáticas $(p=.000)$, Sociales $(p=.000)$, Naturales $(p=.001)$ y Religión $(p=.000)$. El análisis post hoc confirma una relación directamente proporcional entre los niveles de felicidad y las calificaciones académicas.

\section{Discusión y Conclusiones}

Este estudio pone de manifiesto la complejidad a la hora de evaluar determinadas situaciones relacionadas con el ámbito emocional. La consideración de una muestra pluricultural y el contexto en el que se realiza este trabajo, se pueden considerar relevantes. Esto permitirá realizar una aproximación al conocimiento emocional en muestras con características similares.

El principal predictor de cada uno de los dos estados emocionales es el otro. La relación queda demostrada directamente (los totales) o bien a través de uno o varios factores. Si se consideran ambos estados emocionales, se pueden contemplar diferencias estadísticamente significativas, tanto para la Felicidad Total, como para cada uno de los factores. Como se ha venido contemplando a lo largo de los diferentes apartados, el comportamiento de ambas variables consideradas (Felicidad en función de los niveles de Ansiedad) es antagónico. Esta realidad apoya las diferencias encontradas, que indican una relación inversamente proporcional entre estados emocionales. De esta forma, 
parece haber interferencia entre la presencia de estados emocionales positivos y los de carácter negativo (Carballeira et al., 2015), llegándose a situar ambos estados como los polos opuestos de un continuo (Fillo, Simpson, Rholes, \& Kohn, 2015; Moreno-Rosset et al., 2016), que van desde la satisfacción con la vida y la felicidad, hasta situaciones de estrés que pueden desembocar en síntomas de ansiedad (Lawrence, Rothman, Cobb, \& Bradbury, 2012). De esta manera, los sujetos que experimentan menores niveles de ansiedad son los que reflejan niveles superiores en bienestar (Moreno-Rosset et al., 2016). El balance entre estados emocionales positivos y negativos, sería un predictor de la satisfacción vital y de un desarrollo más saludable y positivo, que sería un elemento significativo en la prevención de trastornos emocionales como la ansiedad (MorenoRosset et al., 2016).

La cultura/religión del sujeto es un predictor de la variable Felicidad (dentro de las sociodemográficas), encontrándose también diferencias estadísticamente significativas. El sentido de las mismas vuelve a ser inverso al que se describía para la ansiedad. En esta ocasión, son los musulmanes los que presentan niveles superiores. Se encontraron muchos trabajos que refuerzan la importancia concedida al contexto cultural y sus diferencias sobre el bienestar psicológico (Jia et al, 2010; Quiceno \& Vinaccia, 2015; Restrepo et al., 2011). Se entiende que cualquier la consideración de felicidad está ligada a los valores de cada cultura y que sus determinantes no son iguales en grupos culturales diferentes, dado que dependen de múltiples elementos, desde la satisfacción con los aspectos vitales personales, las relaciones familiares, el trabajo y las relaciones sociales y los valores culturales (Carballeira et al., 2015).

El estatus socio-económico y cultural, actúa también como predictor de la Felicidad. En este sentido, aparecieron diferencias estadísticamente significativas, en Felicidad, así como en los factores Condiciones de vida negativas, Satisfacción con la vida y Condiciones de vida positivas. Otra vez, el sentido de las mismas vuelve a ser el ya comentado. Son los de niveles más elevados los que reflejan puntuaciones superiores en esta variable y sus factores. Esta realidad es similar a la de otros autores (Carballeira et al., 2015; Matijasevic, Ramírez, \& Villada, 2010; Quiceno \& Vinaccia, 2015). En este sentido, no sólo existe una asociación positiva entre ingresos económicos y bienestar, sino que el hecho de percibir que las condiciones económicas están mejorando (Matijasevic et al., 2010) y la ocupación laboral del sujeto (Díaz \& González, 2011) son indicadores de un incremento inmediato en la satisfacción con la vida y de un desarrollo más positivo, siendo también indicativo del éxito escolar.

En Ansiedad, el principal predictor, dentro de las variables sociodemográficas, es la Edad, donde también se registran diferencias significativas. En este caso, las diferencias indican un ascenso desde los 12 hasta los 18 años. A continuación aparece el Género, que actúa como predictor de la Ansiedad. Considerando este factor, se vuelven a encontrar diferencias estadísticamente significativas para los totales, así como todos los factores de la Ansiedad. Las personas pertenecientes al el género femenino reflejan puntuaciones más elevadas en todos los casos. El sentido de estas diferencias coincide con la mayoría de trabajos consultados (Franco, Pérez, \& de Dios, 2014; Gómez-Garibello \& Chaux, 2014; Pulido \& Herrera, 2016). Ante estas diferencias encontradas, se concede mayor credibilidad a aquellas que tienen en cuenta diferencias en los patrones de socialización y la presencia marcada de determinados roles (Pulido \& Herrera, 2016). 
Con respecto al Rendimiento, dentro de las variables sociodemográficas, el primer predictor es el Estatus, dado que se encontraron diferencias significativas, tanto para la Media de Rendimiento como para cada una de las materias. En todos los casos, las calificaciones aumentan a medida que lo hace el estatus socio-económico y cultural. Esta realidad se encontró también en otras experiencias (Pulido \& Herrera, 2016).

Con respecto al Rendimiento en función de la cultura/religión (otra de las variables sociodemográficas predictoras), se comprueban diferencias estadísticamente significativas. Estas se dan en Rendimiento (Media), así como en todas y cada una de las materias. En todos los casos, el grupo de alumnos pertenecientes a la cultura/religión cristiana-occidental obtienen mejores resultados, realidad ya fue descrita en otros trabajos (Cervini et al., 2014; Oropesa et al., 2014) y las diferencias de estatus socioeconómico y cultural (Cervini et al., 2014). A esto hay que añadir las importantes diferencias en cuanto a la lengua materna entre musulmanes y cristianos (Siqués \& Vila, 2014) como importantes justificantes de estas diferencias significativas.

Dentro de las variables sociodemográficas, el último predictor es la edad. Se encontraron diferencias significativas en cuanto al Rendimiento en todas las materias, así como también en la Media del Rendimiento. Los resultados más altos son para el alumnado entre 16 y 17 años, dándose diferencias con el resto de grupos de edad. El rendimiento parece tener relación con la edad, indicándose la existencia de estas relaciones. En esta ocasión, se incide a la relación entre el curso y la edad, para justificar estas diferencias. Por esta razón, se vuelve a mencionar el trabajo de Pulido y Herrera (2016) como ejemplo en el que se pueden ver diferencias considerando esta variable.

Se pone fin a este apartado de discusiones relacionadas con la influencia de la ansiedad sobre el Rendimiento Académico. En esta ocasión, no existe ninguna interacción entre ambas variables. De manera contraria a lo que se esperaba, no se encontraron diferencias significativas en cuanto al Rendimiento en ninguna de las materias. La única excepción es la materia de Matemáticas, siendo inversamente proporcionales a los niveles de ansiedad. Este trabajo no coincide con otros en los que sí se contempla una relación directa entre los niveles de ansiedad y el desarrollo educativo (Contreras et al., 2005). Sin embargo, de una forma más intensa actúa la Felicidad, que aparece como predictora del Rendimiento (sus factores). Se encontraron diferencias significativas en cuanto al Rendimiento también en las materias (Lengua Castellana, Matemáticas, Ciencias Sociales y Naturales, Inglés y Religión). La dirección de estas diferencias sigue un orden lógico, siendo directamente proporcional, lo que coincide con otros trabajos en los que también se encontraron este tipo de diferencias (Salanova et al., 2005; Schaufeli \& Bakker, 2005). Esto hace imprescindible la consideración de las competencias emocionales dentro del ámbito educativo. Trabajando sobre ellas se podrá disponer de una poderosa herramienta para la mejora del rendimiento académico, una constante perseguida dentro de nuestro Sistema Educativo.

\section{Referencias}

Alonso, J., \& Román, J. M. (2014). Nivel sociocultural, prácticas educativas familiares y autoestima de los hijos en edades tempranas. Revista de Investigación Educativa, 32(1), 187-202. doi: https://doi.org/10.6018/rie.32.1.173421 
Caballero-Dominguez, C. C., Suárez-Colorado, Y. P., \& Bruges-Carbonó, H. D. (2015). Characteristics of Emotional Intelligence in a Group of Higher Education Students with and without Suicidal Ideation. Revista CES Psicología, 8(2), 138-155. Recuperado de https://dialnet.unirioja.es/servlet/articulo?codigo=5236645

Carballeira, M., González, J. A., \& Marrero, R. J. (2015). Diferencias transculturales en bienestar subjetivo: México y España. Anales de Psicología, 31(1), 199-206. doi: 10.6018/analesps.31.1.166931

Cervini, R., Dari, N., \& Quiroz, S. (2014). Estructura familiar y Rendimiento Académico en países de América Latina. Los datos del Segundo Estudio Regional Comparativo y Explicativo. Revista Mexicana de Investigación Educativa, 19(61), 569-597. Recuperado de http://www.redalyc.org/articulo.oa?id=14030110010

Contreras, F., Espinosa, J. C., Esguerra, G., Haikal, A., \& Rodríguez, A. (2005). Autoeficacia, ansiedad y rendimiento académico en adolescentes. Perspectivas en psicología 1(2), 183-194 Recuperado de http://www.redalyc.org/articulo.oa?id=67910207

Díaz, F., \& González, M. J. (2011). Subjective and Occupational Well-Being in a Sample of Mexican Workers. Social Indicators Research, 100(2), 273-285.

Fillo, J., Simpson, J. A., Rholes, W. S., \& Kohn, J. L. (2015). Dads doing diapers: Individual and relational outcomes associated with the division of child care across the transition to parenthood. Journal of Personality and Social Psychology, 108, 298-316. doi:/10.1037/a0038572

Franco, N., Pérez, M. A., \& de Dios, M. J. (2014). Relación entre los estilos de crianza parental y el desarrollo de ansiedad y conductas disruptivas en niños de 3 a 6 años. Revista de Psicología Clínica con Niños y Adolescentes, 1(2), 149-156. Recuperado de https://dialnet.unirioja.es/servlet/articulo?codigo $=4742071$

Gómez-Castro, J. L. (1986). “Rendimiento escolar y valores interpersonales: Análisis de resultados en E.G.B. con el cuestionario SIV de Leonardo V. Gordon". Bordón, $262,257-275$.

Gómez-Garibello, C., \& Chaux, E. (2014). Agresión relacional en preescolar: variables cognoscitivas y emocionales asociadas. Universitas Psychologica, 13(2), 565-574. doi:10.11144/Javeriana.UPSY13-2.arpv

Inglés, C. J., La Greca, A. M., Marzo, J. C., García-López, L. J., \& García-Fernández, J. M. (2010). Social Anxiety Scale for Adolescents: factorial invariance and latent mean differences across gender and age in Spanish adolescents. Journal of Anxiety Disorders, 24, 847-855. doi: 10.1016/j.janxdis.2010.06.007

Jia, Z., Tian, W., He, X., Liu, W., Jin, C., \& Ding, H. (2010). Mental health and quality of life survey among child survivors of the 2008 Sichuan earthquake. Quality of Life Research, 19, 1381-1391. doi: 10.1007/s11136-010-9703-8.

Lawrence, E., Rothman, A. D., Cobb, R. J., \& Bradbury, T. N. (2012). Marital satisfaction across the transition to parenthood: Three eras of research. Washington, DC: American Psychological Association.

Matijasevic, M. T., Ramírez, M., \& Villada, C. (2010). Bienestar subjetivo: Una re-visión crítica de sus resultados, alcances y limitaciones. Regiones, 5(1), 5-39.

Moreno-Rosset, C., Arnal-Remón, B., Antequera-Jurado, R., \& Ramírez-Uclés, I. (2016). Anxiety and psychological wellbeing in couples in transition to parenthood. Clínica y Salud, 27, 29-35. doi:10.1016/j.clysa.2016.01.004 
Oropesa, F., Moreno, C., Pérez, P., \& Muñoz-Tinoco, V. (2014). Rutinas de tiempo libre: oportunidad y riesgo en la adolescencia. Cultura y Educación, 26(1), 170-183. doi: https://doi.org/10.1080/11356405.2014.908670

Pulido, F., \& Herrera, F. (2016). Miedo y rendimiento académico en el contexto pluricultural de Ceuta. Revista de Investigación Educativa, 34(1), 185-203. doi: http://dx.doi. org/10.6018/rie.34.1.207221

Quinceno, J. M., \& Vinaccia, S. (2015). Calidad de vida, fortalezas personales, depresión y estrés en adolescentes según sexo y estrato. International Journal of Psychology and Psychological Therapy, 14(2), 155-170. Recuperado de http://www.redalyc.org/articulo. oa?id=56031293002

Ranoyen, I., Jozefiak, T., Wallander, J., Lydersen, S., \& Indredavik, M. S. (2014). Selfreported social anxiety symptoms and correlates in a clinical (CAP) and a community (Young-HUNT) adolescent sample. Social Psychiatry and Psychiatric Epidemiology, 49(12), 1937-1949.

Restrepo, C., Vinaccia, S., \& Quiceno, J. M. (2011). Resilencia y depressión: Un estudio exploratorio desde la calidad de vida en la adolescencia. Suma Psicológica, 18, 41-48. Recuperado de http://www.redalyc.org/articulo.oa?id=134222985004

Rodríguez, C. (2010). Variables psicológicas asociadas con la felicidad en centros periurbanos y urbanos marginales de Lima. UCV-Scientia, 2(1), 61-68. Recuperado de https://dialnet.unirioja.es/servlet/articulo?codigo $=6181501$

Salanova, M., Martínez, I. M., Bresó, E. Llorens, S., \& Grau, R. (2005). Bienestar psicológico en estudiantes universitarios: facilitadores y obstaculizadores del desempeño académico. Anales de psicología, 21(1), 170-180. Recuperado de http://www.redalyc. org/articulo.oa?id=16721116

Schaufeli, W. B., \& Bakker, A. B. (2005). Job demands, job resources and their relationship with burnout and engagement: A multi-sample study. Journal of Organizational Behavior, 25, 293-315. Recuperado de https:/www.wilmarschaufeli.nl/publications/ Schaufeli/209.pdf

Siqués, C., \& Vila, I. (2014). Estrategias discursivas y aprendizaje de la lengua escolar: un estudio etnográfico en un aula de alta diversidad étnica y lingüística. Cultura y Educación, 26(2), 349-376. doi: https://doi.org/10.1080/11356405.2014.935112

Spielberger, C. D. (2001). STAIC. Cuestionario de Ansiedad Estado/Rasgo en niños. Madrid: TEA.

SPSS (2011). IBM SPSS Statistics for Windows, Version 20.0. Armonk, NY: IBM Corp.

Fecha de recepción: 27 de marzo de 2017.

Fecha de revisión: 19 de abril de 2017.

Fecha de aceptación: 29 de septiembre de 2018. 
